\title{
L0 Gradient based Image Smoothing Method for Ear Identification
}

\author{
Ying Tian ${ }^{1}$ and Haodi $\mathrm{Ma}^{2}$ \\ ${ }^{1,2}$ University of Science and Technology Liaoning \\ Anshan, 114051, China \\ astianying@126.com, mahaodi_0123@163.com
}

\begin{abstract}
Ear identification is an important biometric identification technique and has been widely used in many applications. One of the most important components in ear identification system is image preprocessing. The performance of image preprocessing has a significant impact on the accuracy of ear identification system. This paper proposes a LO gradient based image smoothing method for ear identification. First, ear images are filtered using LO gradient based method. Then the contrast of the image is enhanced using histogram equalization method in order to make ear edges more discriminative. Comparative experiments with an existing algorithm demonstrate that our method has better performance and is more suitable for ear identification.
\end{abstract}

Keywords: L0 gradient, Image preprocessing, Ear identification

\section{Introduction}

Human body biometric identification technology is very important in many scientific and engineering areas. One of the most commonly used techniques is ear identification. The first ear identification system was presented by Alfred lannarelli. He has studied approximately 1000 people's ears and approved that the physiological structure of human ears are different between each other. Then a lot of researchers improved this technique [1-6]. For example, in, the authors proposed an automatic ear identification algorithm based on Voronoi graph method. Yuizono et al. utilized genetic algorithm to id different ears.

In early times, the ear identification algorithms were mostly based on two dimensional (2D) images [1-2, 7-8]. While, recently, some researchers have utilized three dimensional (3D) structures to perform ear identification [6,9]. There are also algorithms that use both 2D and 3D data [10]. Usually, an ear identification system has five components: image capturing, image preprocessing, feature extraction, pattern recognition, and giving the identification results. Features used in ear identification systems contain SIFT (scale invariant feature transform) features [2, 11], ASIFT (Affine SIFT) features [12, 13] et al..

Image preprocessing is much crucial for ear identification systems. Because ear images captured by cameras have a lot of noise, it is necessary to smooth the images before the feature extraction procedure. The preprocessing result has a significant impact on the accuracy of ear identification algorithms. The most commonly used image smoothing techniques are Gaussian smoothing and bilateral filtering. Gaussian filter is a kind of linear filter, while bilateral filter is a non-linear filter. Both of them have been widely used in a variety of image processing and computer vision applications [14-18]. Gaussian filter has good performance on image smoothing. However, the edge information of objects in the image is also reduced because the Gaussian smoothing is a process of weighted average carried out on the whole image. Bilateral filter is an edge-preserving smoothing technique, but it makes a trade off between edge preservation and detail flattening. Therefore the accuracy of the ear identification system will not be satisfied 
after Gaussian smoothing or bilateral smoothing. In 2011, Xu et al. [19] proposed a novel image smoothing technique via L0 gradient minimization. This technique can sharp major edges of objects in the image by increasing the steepness of transition. Meanwhile, it can also eliminate noise and insignificant structures.

In this paper, proposed an ear identification system using the L0 smoothing method. Comparative experimental results demonstrate that our algorithm has better performance than algorithms based on Gaussian smoothing and bilateral smoothing.

The reminder of this paper is organized as follows. Section 2 explains the image preprocessing procedure in details. Section 3 shows the experiments carried out on an existing ear image dataset. Conclusions and future work are presented in section 4 .

\section{Image Preprocessing based on LO Smoothing}

Image preprocessing is an important component for ear identification systems. In this section they will describe this procedure in details. First, they smooth the image using L0 gradient method. Then the contrast of the image is enhanced to make the ear more discriminative.

\subsection{L0 Gradient based Image Smoothing}

L0 gradient based image smoothing is a useful technique for image processing, which smoothes the image in a global manner. Highest-contrast edges are enhanced by confining the number of non-zero gradients.

Assume $I$ and $S$ are the input image and smoothed image, respectively. Let $P$ be a pixel in image $S$. The gradient at pixel $P$ can be presented as

$\nabla S_{p}=\left(\partial_{x} S_{p}, \partial_{y} S_{p}\right)^{T^{\nabla}}$

Where $\partial_{x} S_{p}$ and $\partial_{y} S_{p}$ are calculated by measuring the difference between neighboring pixels along $x$ and $y$ coordinate directions. Then the gradient measure can be expressed as

$$
C(S)=\#\left\{p|| \partial_{x} S_{p}|+| \partial_{y} S_{p} \mid \neq 0\right\}
$$

It counts the number of pixels where the magnitude $\left|\partial_{x} S_{p}\right|+\left|\partial_{y} S_{p}\right|$ is not zero.

With Eq. (1) and Eq. (2), they can compute the smoothed image by solving the following optimization problem.

$$
\min _{S}\left\{\sum_{p}\left(S_{p}-I_{p}\right)^{2}+\lambda \cdot C(S)\right\}
$$

Where $\lambda$ is a user defined constant.

This optimization problem is intractable and can not be solved by using commonly used methods, such as gradient decent method. Therefore it is necessary to bring in two auxiliary variables $h_{p}$ and $v_{p}$ to approximate the optimized solution using an iterative method, where $h_{p}$ is introduced for $\partial_{x} S_{p}$ and $v_{p}$ is introduced for $\partial_{y} S_{p}$. Then the optimization problem in Eq. (3) can be rewritten as follows

$$
\min _{S, h, v}\left\{\sum_{p}\left(S_{p}-I_{p}\right)^{2}+\lambda C(h, v)+\beta\left(\left(\partial_{x} S_{p}-h_{p}\right)^{2}+\left(\partial_{y} S_{p}-v_{p}\right)^{2}\right)\right\}
$$

In this equation, $\beta$ is an automatically tuned variable, which can control the similarity between $(h, v)$ and their gradients. When $\beta$ gets larger enough, Eq. (4) is equal with Eq. (3). $C(h, v)$ is similar with $C(S)$ in Eq. (2) and can be computed by 


$$
C(h, v)=\#\left\{p|| h_{p}|+| v_{p} \mid \neq 0\right\}
$$

Now they can estimate $S$ and $(h, v)$ separately. Given $(h, v)$, they can compute $S$ by solving the following sub-problem

$$
\min _{S}\left\{\sum_{p}\left(S_{p}-I_{p}\right)^{2}+\beta\left(\left(\partial_{x} S_{p}-h_{p}\right)^{2}+\left(\partial_{y} S_{p}-v_{p}\right)^{2}\right)\right\}
$$

Similarly, given $S,(h, v)$ can be computed by solving the objective function as

$$
\min _{h, v}\left\{\sum_{p}\left(\partial_{x} S_{p}-h_{p}\right)^{2}+\left(\partial_{y} S_{p}-v_{p}\right)^{2}+\frac{\lambda}{\beta} C(h, v)\right\}
$$

Both of the sub-problems described in Eq. (6) and Eq. (7) have closed-form solutions. Details of the solving method can be found in.

In the solving of the overall optimization problem, parameter $\lambda$ can be tuned to control the level of structure coarseness. At the beginning of iteration, parameter $\beta$ is set to a small value. Then it will be multiplied by user defined parameter $\kappa$ at each iteration. This can speed up the convergence according to [20].

\subsection{Image Contrast Enhancing}

In the process of imaging, transferring, or transforming, the contrast of the image may usually be decreased. According to our implementation, the contrast of the ear images is quite low after L0 smoothing and not suitable for used directly. Therefore, they should enhance its contrast in this preprocessing procedure. In this paper, they use the histogram equalization method to enhance image contrast.

Assume the image has totally $N$ pixels and $L$ gray levels. Let $r_{k}$ be the value of the $k^{\text {th }}$ gray level in the image and $n_{k}$ be the number of pixels that have the value of $r_{k}$. Then the frequency of pixels that have the value of $r_{k}$ can be calculated by

$$
P_{r}\left(r_{k}\right)=n_{k} / N \quad\left(0 \leq r_{k} \leq 1, k=0,1 \cdots L-1\right)
$$

Finally they obtain the transformed gray level $S_{k}$ corresponding to $r_{k}$ :

$$
S_{k}=\sum_{j=0}^{k} P_{r}\left(r_{j}\right)=\sum_{j=0}^{k} \frac{n_{j}}{N}
$$

After the histogram equalization, they get an image that has high contrast and suitable for ear identification.

\section{Main Title}

In this section, they will present some comparative experiments to verify the proposed algorithm. The images used in this paper are downloaded from some widely used datasets. First, experiment on a single ear image was done to demonstrate that our preprocessing method outperforms the existing method. Then, ear identification was carried out on a set of images to verify that the preprocessing procedure has a significant impact on ear identification. Using our algorithm, they can get higher accuracy.

\subsection{Image Preprocessing}

The image used in this experiment was chosen from the USTB ear image dataset [21]. The original image is shown in Figure 1. First, they smoothed the image using Gaussian method, bilateral method, and the L0 method, respectively. Then the images were processed to enhance contrast via histogram equalization. Finally, edge extraction was 
carried out on these images to compare the performances of these three methods. Here, the user defined parameters $\lambda$ and $\kappa$ are set to 0.005 and 8.0, respectively.
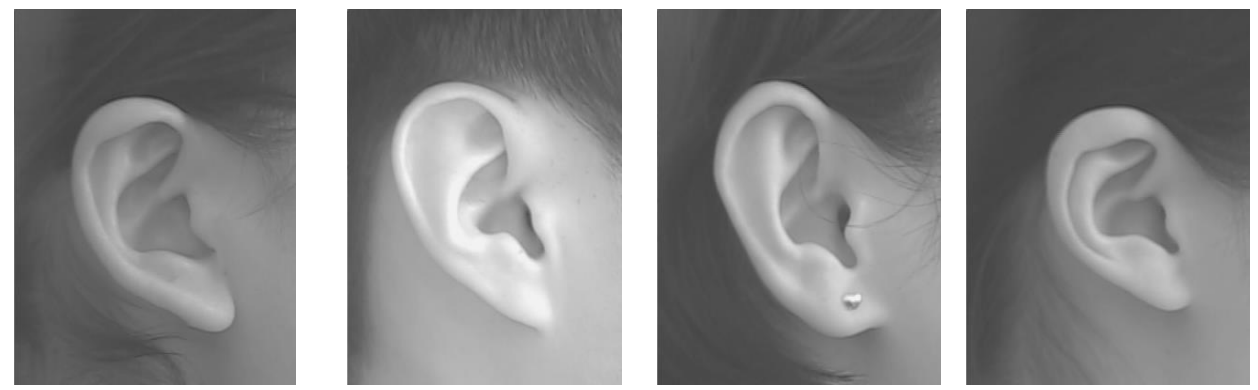

Figure 1. Original Image Chosen from USTB Dataset

Such as the first image. The processing results can be seen in Figure 2. Results of Gaussian method, bilateral method, and L0 method are shown in the first, second, and last rows, respectively. Figure 2(a), 2(d), and 2(g) present the smoothing results. Figure 2(b), 2(e), and 2(h) show the images after contrast enhancement. From Figure 2(a) and 2(b), they can see that the edges of the ear are blurred when using Gaussian smooth technique. While according to Figure 2(g) and 2(h), they can see that the useless noise and structures are eliminated. But the edges of the ear still exist and are very sharp. At this step, the smoothing results of bilateral method and $\mathrm{L} 0$ method are much similar in appearance. One of the differences can be seen at the hair on the temples, as shown in Figure 2(e) and 2(h). Larger differences appear at next step. The edge extraction results are presented in Fig. 2(c), 2(f), and 2(i). As shown in these figures, the edges of Gaussian method are incomplete. The result of bilateral method is better, but is still not good enough. As Figure 2(i) shows, the edges of L0 method are almost lossless.

More results can be seen in Figure 3. The last column presents the processing results using L0 based algorithm. The results of this experiment demonstrate that L0 gradient based image smoothing technique is more suitable for ear identification and our preprocessing algorithm has better performance.

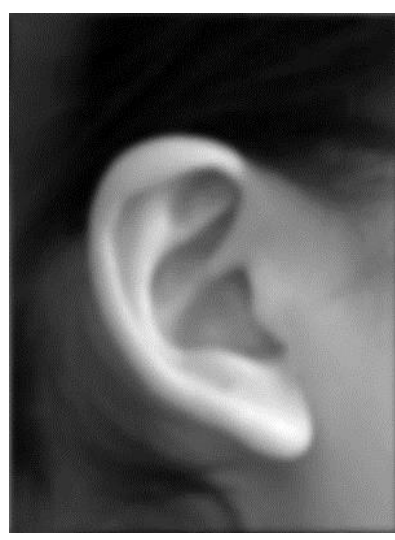

(a)

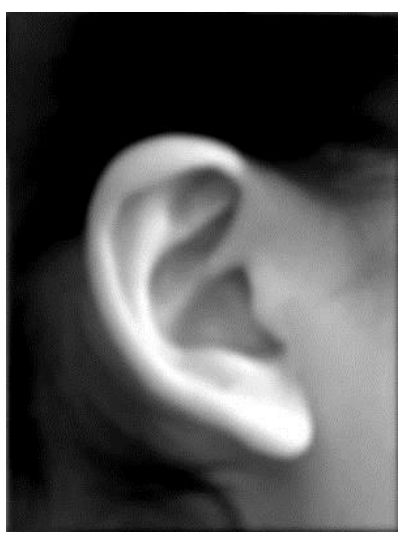

(b)

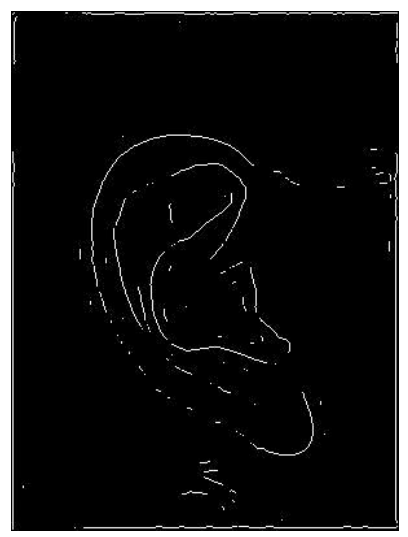

(c) 


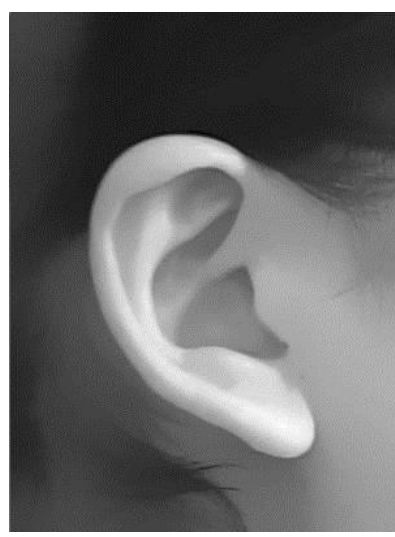

(d)

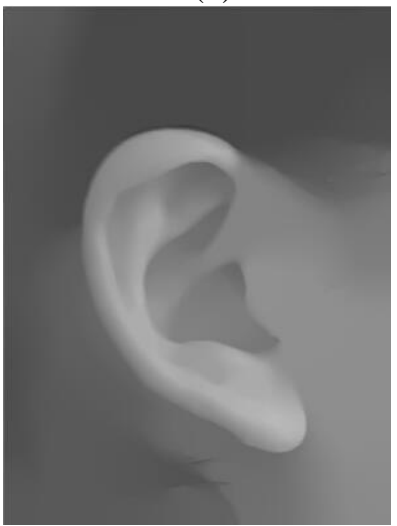

(g)

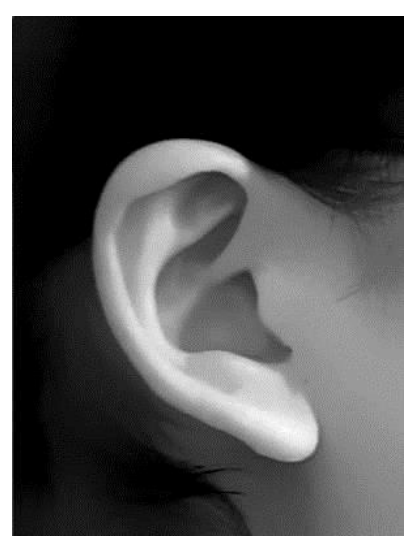

(e)

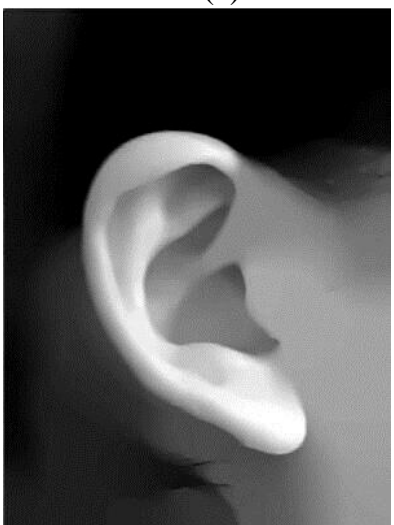

(h)

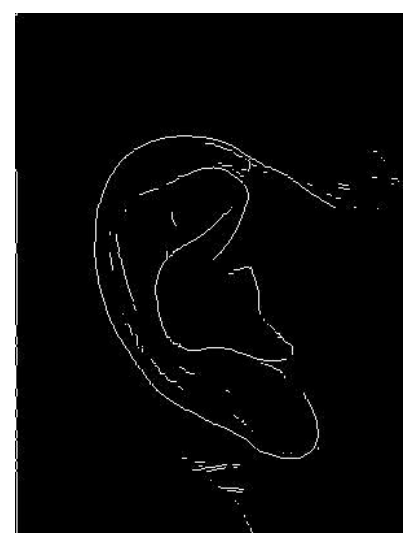

(f)

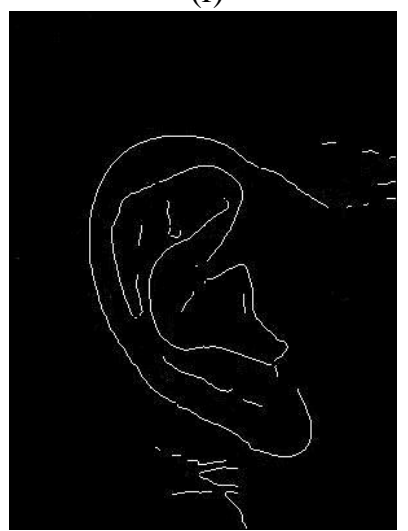

(i)

Figure 2. Processing Results of Different Methods; Results of Gaussian Method, Bilateral Method, and LO Method are Shown in First, Second, and Last Rows, Respectively; (a, d, g) Smoothing Results; (b, e, h) Results after Contrast Enhancement; (c, f, i) Edge Extraction Results
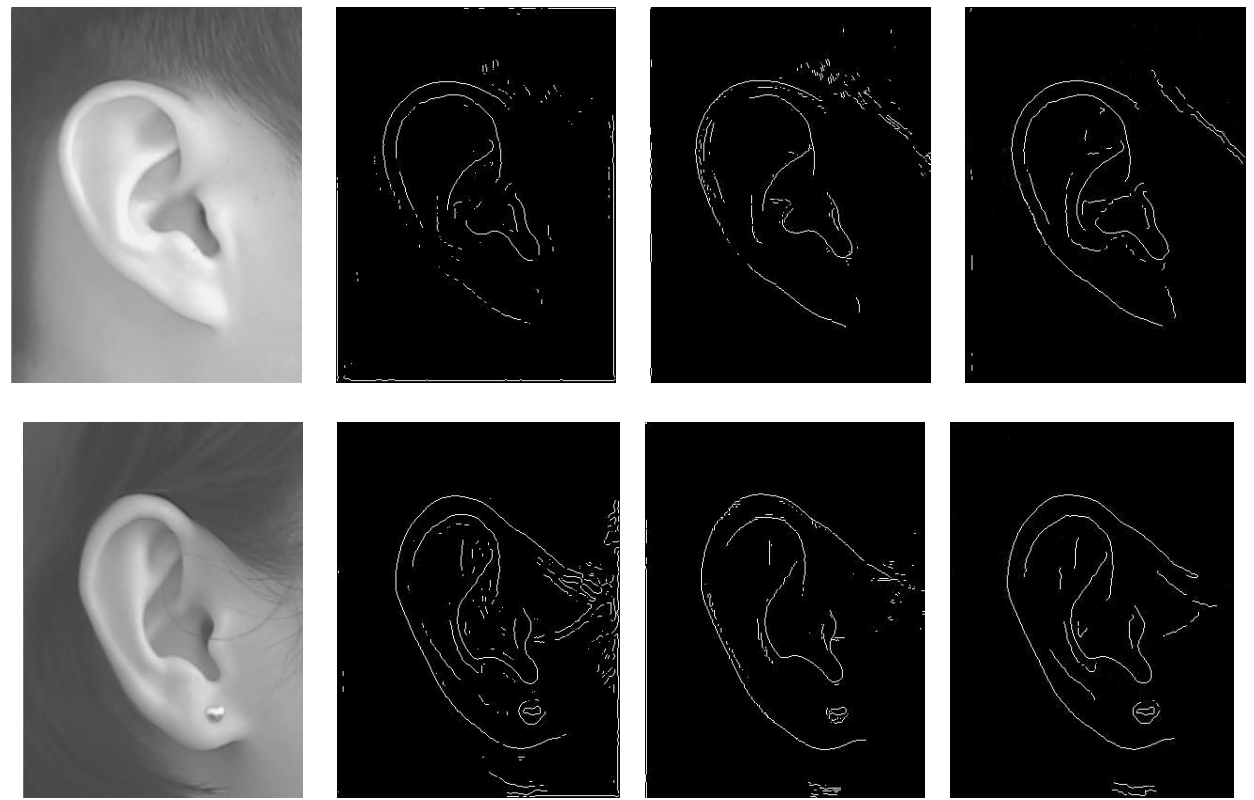

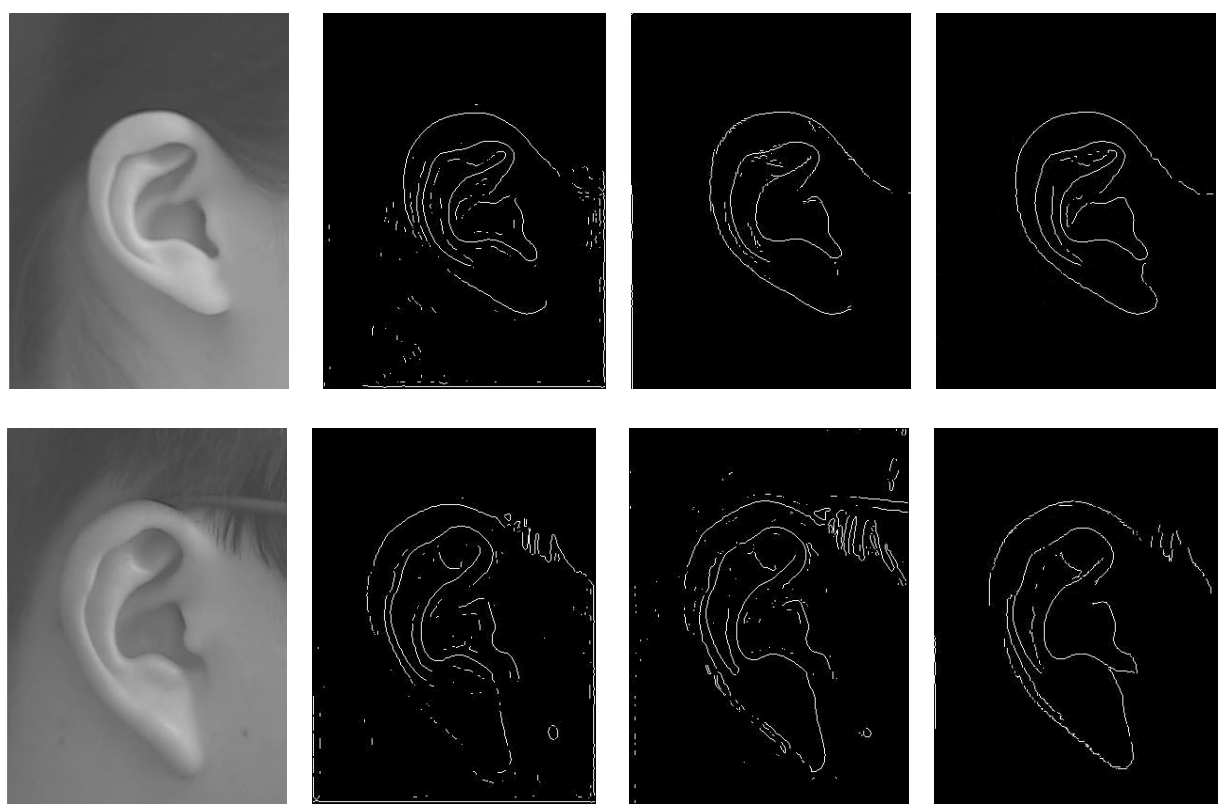

Figure 3. Preprocessing Results of Different Methods; The First Column Shows the Original Ear Images; Processing Results of the Gaussian based, Bilateral based, and LO based Algorithms are Presented in the Second, Third, and Fourth Columns, Respectively

\subsection{Ear Identification}

In this subsection, to further verify the effectiveness of L0 smoothing for ear identification, they present the results of ear identification carried on a set of datasets. Besides the USTB dataset discussed above, XM2VTS dataset [22], UND2D dataset [23], and FERET dataset [24] were also utilized. Since some of the datasets are the collections of side face images, they first cut out ear areas manually.

Note that the focus of this paper is image preprocessing for ear identification, the classification or recognition methods are not concerned. The aim of this experiment is to demonstrate that the proposed preprocessing algorithm is more suitable for ear identification systems. Therefore the classification algorithm presented in is used, which has mainly five steps: contour detection, binarization, coordinates normalization, feature extraction, and finally classification.

In each dataset, they select 100 groups of different ear images. Each group consists of 5 images from the same person, in which 1 as testing image and 4 as training images. So they have 100 testing images and 400 training images for each dataset. The ratio of the number of correctly recognized images to the number of total groups is recorded as the accuracy for each dataset. The results are presented in Tab. 1. More intuitive comparison is illustrated in Figure 4, in which identification rates of L0 based algorithm, bilateral based algorithm, and Gaussian based algorithm are represented by solid line with dots, dash-dot line with circles, and dot line with asterisks, respectively. The results show that no matter which dataset is used, our method has the best accuracy.

\section{Table 1. Ear Identification Rates using different Methods on Different Datasets}

\begin{tabular}{lcccc}
\hline Method & $\begin{array}{c}\text { USTB } \\
\text { dataset }\end{array}$ & $\begin{array}{c}\text { XM2VTS } \\
\text { dataset }\end{array}$ & UND2D dataset & FERET dataset \\
\hline Gaussian based & 0.93 & 0.87 & 0.89 & 0.90 \\
Bilateral based & 0.95 & 0.90 & 0.94 & 0.93 \\
L0 based & 0.98 & 0.95 & 0.96 & 0.95 \\
\hline
\end{tabular}




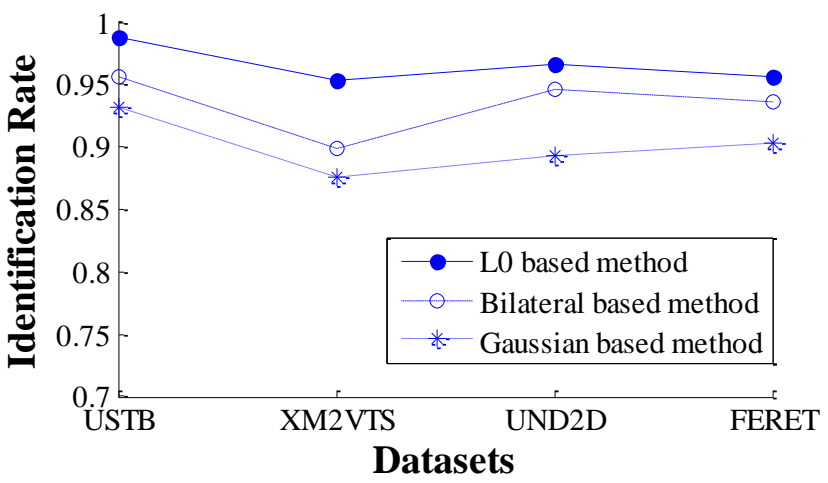

Figure 4. Ear Identification Rates Comparisons; Results of LO based, Bilateral based, and Gaussian based Methods are Illustrated by Solid Line with Dots, Dash-dot line with Circles, and Dot Line with Asterisks, Respectively

\section{Conclusions}

This paper proposed a L0 gradient based image smoothing method for ear identification. Different with Gaussian smoothing and bilateral smoothing et al. commonly used image preprocessing techniques, L0 gradient based image smoothing can eliminate useless noise and structures while reserve useful edges in the mean time. Experiments on ear images preprocessing demonstrate that L0 based smoothing outperforms Gaussian based and bilateral based smoothing. After image contrast enhancement, they can extract ear edges exactly. Ear identification experiments on some widely used datasets were also carried out. The results show that they can make a significant increase in correct recognition rate using our method.

According to our experiments, they find that the proposed preprocessing algorithm has the same limitation with other methods. That is if the ear images are captured under different lighting conditions, the identification rate of the ear identification system will drop heavily. Therefore they would like to improve our method to overcome this limitation in the future.

\section{References}

[1] T. Yuizono, Y. Wang, K. Satoh, S. Nakayama, Study on Individual Recognition for ear images by using genetic local search. Proceedings of 2002 Congress Evolutionary Computation, (2002), pp. 237-242.

[2] J. D. Bustard, M. S. Nixon. Robust 2D ear registration and recognition based on SIFT point matching. 2th IEEE International Conference on Biometrics: Theory, Applications and Systems, (2008), pp. 1-6.

[3] L. Yuan, Z. C. Mu and Y. Zhang, "Ear recognition with occlusion based on improved nonnegative matrix factorization with sparseness constraint", Proceedings of the 18th International Conference on Pattern Recognition, Hong Kong, (2006), pp. 1-6.

[4] I. Naseem, R. Togneri and M. Bennamoun, "Sparse representation for ear biometrics", Proceedings of the 4th International Symposium on Advances in Visual Computing, Part II, Las Vegas, NV, (2008), pp. 336-345.

[5] D. Hurley, M. Nixon and J. Carter, "Force field feature extraction for ear biometrics", Computer Vision and Image Understanding, vol. 98, no. 3, (2005), pp. 491-512.

[6] P. Yan and K.W. Bowyer, "Biometric recognition using 3D ear shape. IEEE Transactions on Pattern Analysis and Machine Intelligence, vol. 29, no. 8, (2007), pp. 1297-1308.

[7] M. Choras, "Ear biometrics based on geometrical feature extraction", Electronic Letters on Computer Vision and Image Analysis, vol. 5, no. 3, (2005), pp. 84-95.

[8] S. Z. Wang, "An improved normalization method for ear feature extraction”, Int. J. Signal Process. Image Process. Pattern Recogn., vol. 6, no. 5, (2013), pp. 49-56. 
[9] B. Bhanu and H, Chen, "Human ear recognition in 3D", Workshop on Multimodal User Authentication, (2003), pp. 91-98.

[10] P. Yan and K. W. Bowyer, "Ear biometrics using 2D and 3D images", Proc. 2005 IEEE CS Conf. Computer Vision and Pattern Recognition - Workshops, (2005), pp. 121.

[11] D. G. Lowe, "Distinctive image features from scale-invariant key points", International Journal of Computer Vision, vol. 60, no. 2, (2004), pp. 91-110.

[12] Y. Tian, C. Xu, Y.M. Zhang. Ear recognition based on Gabor wavelet and ASIFT feature points. Computer Applications and Software, vol. 30, no. 11, (2013), pp. 202-205.

[13] J. Morel and G. Yu, "ASIFT: A new framework for fully affine invariant image comparison", SIAM Journal on Imaging Sciences, vol. 2, no. 2, (2009).

[14] A. Mousa, "Canny edge-detection based vehicle plate recognition", Int. J. Signal Process. Image Process. Pattern Recogn., vol. 5, no. 3, (2012), pp. 1-8.

[15] X. Pennec, P. Fillard and N. Ayache, "A Riemannian framework for tensor computing", International Journal of Computer Vision, vol. 66, no. 1, (2006), pp. 41-66.

[16] Z. Farbman, R. Fattal, D. Lischinski and R. Szeliski, "Edge-preserving decompositions for multi-scale tone and detail manipulation", ACM Transactions on Graphics, vol. 27, no. 3, article 67, (2008).

[17] Q. X. Yang, "Hardware-efficient bilateral filtering for stereo matching", IEEE Transactions on Pattern Analysis and Machine Intelligence, vol. 36, no. 5, (2014), pp. 1026-1032.

[18] A. Gabiger-Rose, M. Kube, R. Weigel and R. Rose, "An FPGA-based fully synchronized design of a bilateral filter for real-time image denoising", IEEE Transactions on Industrial Electronics, vol. 61, no. 8, (2014), pp. 4093-4104.

[19] L. Xu, C. Lu, Y. Xu and J. Y. Jia, "Image smoothing via L0 gradient minimization", ACM Transactions on Graphics, vol. 30, no. 6, (2011), Article 174.

[20] Y. Wang, J. Yang, W. Yin and Y. Zhang, "A new alternating minimization algorithm for total variation image reconstruction", SIAM Journal on Imaging Sciences, vol. 1, (2008), pp. 248272.

[21] http://www1.ustb.edu.cn/resb/.

[22] http://www.ee.surrey.ac.uk/CVSSP/xm2vtsdb/.

[23] http://www.nd.edu/ cvrl/UNDBiometricsDatabase.html.

[24] P. J. Phillips, H. Moon, S. A. Rizvi and P. J. Rauss, "The FERET evaluation methodology for face recognition algorithms", IEEE Transactions on Pattern Analysis and Machine Intelligence, vol. 22, (2000), pp. 1090-1104.

\section{Authors}

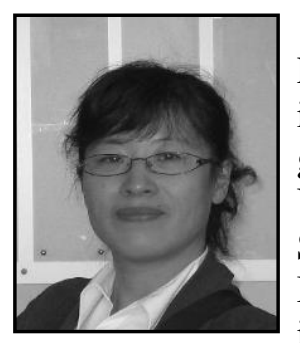

Ying Tian, she has received Ph.D. degrees on Digital Image Processing from the Shenyang University of Technology, 2008. She is Doctor of Computer Science, professor and supervisor of post graduate in University of Science and Technology Liaoning University studies: Measurement technology and instrument in Shenyang University of Technology Scientific interest: Biometrics, Pattern recognition and Image processing. Now she is a professor at University of Science and Technology Liaoning, Anshan, China. She mainly focuses on pattern recognition and digital image processing.

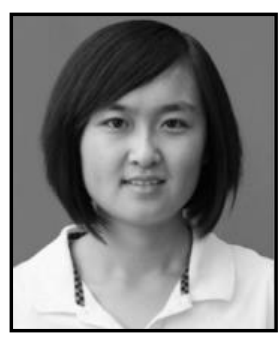

Haodi Ma, she has received B.S degrees in Software institute software engineering from the University of Science and Technology Liaoning, 2012.Now, she is a graduate school student in University of Science and Technology Liaoning University. She mainly focuses on studies: pattern recognition and digital image processing. 